\title{
Research and Application on BP Neural Network Algorithm
}

\author{
Zhao Yan \\ Bohai University, Jinzhou, P.R. China \\ zhaoyan228@126.com
}

Keywords: neural network; BP algorithm; basic idea; model; improvement; application

\begin{abstract}
In the artificial neural network, BP neural network is a multilayer feedforward neural network which is used widely. BP neural network uses a classic BP algorithm, and it is in accordance with the error back-propagation algorithm for learning and training. This paper first analyzes the basic idea of BP algorithm, and using BP neural network model and the flow chart to illustrate; then, introduces the disadvantage of BP algorithm, has slow convergence and easy to fall into local minimum point and other defects, and describes the current improvements methods, such as adding momentum item, introduce variable step method and other optimization methods, which effectively improve the convergence of BP algorithm, to avoid falling into local minimum point; finally, describes in detail the application of BP neural network in face recognition. Effective research on BP neural network, which can be further development and application of BP networks play an important role.
\end{abstract}

\section{Introduction}

Artificial neural network (ANN), also known as neural networks (NN), is composed of a large number of processing elements (neurons) made extensive interconnection network, is the abstraction, simplification and simulation of human brain, which reflects the basic characteristics of the human brain, which is similar to the human brain can be summarized in two aspects: through the learning process using neural network to acquire knowledge from external environment; internal neurons (synaptic weights) used to store acquire knowledge and information [1]. Artificial neural networks is a branch of artificial intelligence science developed rapidly in recent years, is widely used in recent years once again demonstrated its active vitality. BP neural network in the field of neural networks is currently one of the most widely used models, which is one of feedforward networks most commonly used network can achieve the mapping transformation.

BP (back propagation) neural network is also known as multi-layer feedforward network, which is in accordance with the error back-propagation algorithm for learning and training, it does not require prior knowledge about the mathematical equation of expression mapping, will be able to train and store large amounts of input and output mode mapping relationship [2]. Because BP neural network uses a classic BP algorithm, while BP algorithm is based on gradient steepest descent method with squared error as the objective function, which enables the use of neural network algorithms have the ability of learning and memory. And in theory, BP neural network can approximate any continuous nonlinear function: a simple three layer BP neural network can achieve any one from $\mathrm{n}$ dimension to $\mathrm{m}$ dimensional mapping, and its thinking clear, easy programming, simple structure, high precision, strong operability, so it has been very widely used in many fields. The main application fields have pattern recognition, intelligent control, fault diagnosis, image recognition processing, optimal calculation, information processing, financial forecasting, market analysis and business management, and so on.

\section{The Basic Idea of BP Algorithm}

BP neural network has not only the nodes in input layer, output layer node, and there are one or more layer hidden nodes. For input information, the first forward spread to the nodes of the hidden layer, through the activation function of each unit (also known as action function, conversion function) to operate, after the operation, the output information of hidden node disseminate to the 
output node, and finally gives the output results. BP neural network model is shown in Fig. 1. BP network uses a gradient descent method, gradient descent method is based on the gradient of the error function for every two nodes weights, and calculate the weight contribution of the error function, and then according to the gradient information to modify the weights in order to achieve the purpose of learning. BP network can have multiple hidden layers, with h hidden layer, according to feedforward order, the hidden layer nodes is $m_{1}, m_{2} \ldots m_{h}$; each hidden layer output is $y_{1}, y_{2} \ldots y_{h}$; weight matrix for each layer is $w_{1}, w_{2} \ldots w_{h+1}$, then each weight adjustment formula is: output layer: $\quad \Delta \mathrm{w}_{j k}^{h+1}=\eta \delta_{j}^{h+1} \mathrm{y}_{j}^{h}=\eta\left(d_{k}-o_{k}\right) o_{k}\left(1-o_{k}\right) y_{j}^{h}, j=0,1,2, \ldots m_{h} ; k=1,2, \ldots n \quad, \quad h \quad$ hidden layer: $\Delta \mathrm{w}_{i j}^{h}=\eta \delta_{j}^{h} \mathrm{y}_{j}^{h-1}=\eta\left(\sum_{k=1}^{n} \delta_{k}^{o} w_{j k}^{h+1}\right) y_{j}^{h}\left(1-y_{j}^{h}\right) y_{j}^{h-1}, i=0,1,2, \ldots m_{h-1} ; j=1,2, \ldots m_{h}$, According to the rule to analogize can obtain the first layer weights adjustment calculation formula [3].

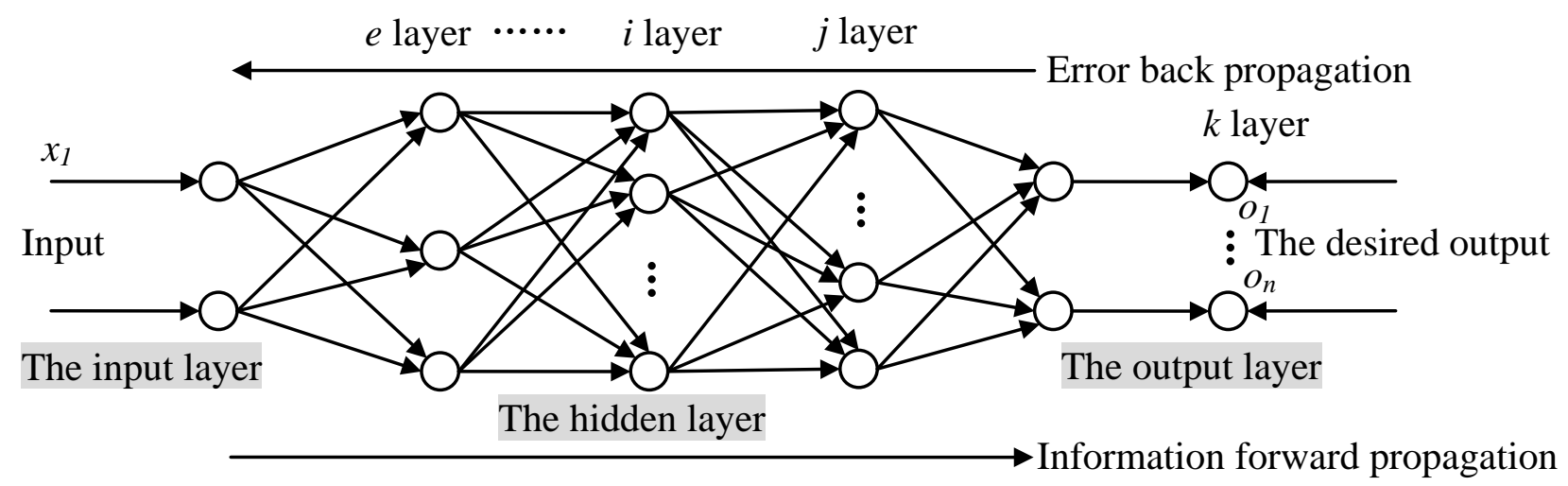

Fig . 1. BP neural network model

The main idea of BP algorithm is the learning process into signal forward propagation and error back propagation in two stages [4]. In the forward propagation stage, the input information from the input layer through the hidden layer to the output layer is transmitted, generating an output signal at an output terminal. In the process of the signal passed along the network weight value is fixed, the state of each layer neuron only affects the lower layer neuron state. If the desired output cannot get in the output layer, there is an error between the actual outputs value and the desired output value, and then turned back propagation process. In the back-propagation phase, error signal returns along the original connection path, by modifying the weights of each layer neurons, successively to the input layer propagation to calculate, and then through the forward propagation process, repeated use of these two processes, such that the error signal is minimized. In fact, when error reached the desired requirements, the network learning process is over. BP algorithm flow chart is shown in Fig. 2.

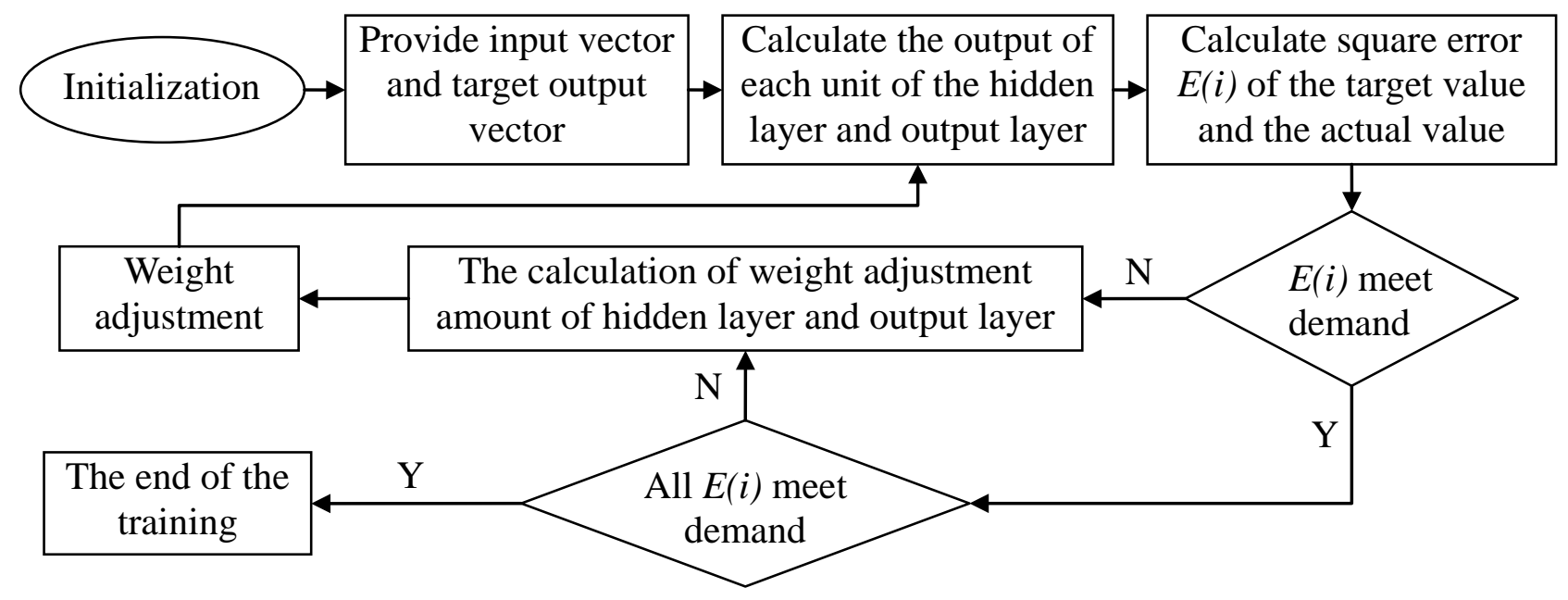

Fig . 2. BP algorithm flow chart 


\section{Inadequate and Improved on BP Algorithm}

Although BP network with nonlinear capabilities, generalization ability and strong fault tolerance ability, but it also has many deficiencies. BP network is slow convergence speed, easily falling into local minimum. BP network using gradient descent method, training from a starting point of the slope gradually reaches the minimum error. For complex networks, the error function is curved multi-dimensional space, which in the process of training may fall into a small valley area and generate local minima, so that training cannot jump out of the local minimum value [5].

Network structure is difficult to determine, the structural parameters (including hidden layers numbers, hidden layer neurons numbers) lack of theoretical guidance, usually only be selected based on experience; poor generalization ability, under normal circumstances, training ability is poor, the generalization ability is also poor, and to a certain extent, with the training ability to improve, generalization ability is improved. But this trend has a limit, when this limit is reached, with the training ability to improve, generalization ability declines and that the so-called "over-fitting" phenomenon. At this point, the network learning too many details of the sample, which does not reflect that the samples contains the rules [6].

At present, the BP algorithm has been obtained for a number of improved methods, which comprises adding momentum item, that is based on the original adds an additional momentum, namely $\Delta \mathrm{w}_{j i}(a+1)=\eta\left(\mathrm{c}_{1} v(a)+\mathrm{c}_{1} v(a-1)\right)$, which $v(a)$ represents a negative gradient in $a$ moment, $\eta$ is the learning rate, $c_{1}$ and $c_{2}$ is the momentum factor; the adoption of better initial weights; function output limit method; variable step method and so on [7]. As the error function improved, can effectively avoid the neurons output fall into the saturated zone of Sigmoid type function, improve the learning speed; eliminates local minimum; at the same time be able to raise the rate of adaptive learning algorithm, but must first be selected some parameters can change the learning rate in the learning training. The learning rate $\eta$ has a greater impact on the convergence speed of the parameter, $\eta$ get bigger, more dramatic changes in the weights, the faster convergence, but easy to make learning and training process oscillation, $\eta$ get smaller, weights change is the smaller, but also make the convergence rate of decline. In order to learn training as much as possible to speed up the convergence rate, should select the appropriate learning rate. To solve this problem, usually under the premise of ensuring convergence precision, as far as possible to improve the $\eta$ value, accelerated the convergence rate.

\section{Application of BP Neural Network}

Face recognition is a typical problem in the field of pattern recognition and image analysis, face recognition technology as an authentication technology is widely used in finance, security and other fields. However, under different light, different acquisition angles, different expressions, these factors have a great influence on the face recognition. The same person's face features exhibit very different, for face recognition to cause great difficulties. Therefore, improving the robustness of face recognition has become an important topic in the field of pattern recognition [8].

For face recognition, is one of the greatest human visual function, neural networks inspired from the nervous system of animals, the use of a large number of simple processing units which constitute the complex interconnected systems to solve complex problems of pattern recognition and behavior control. The BP neural network is used in face recognition, face recognition model is established, through the implementation of image compression, image sampling and standardization of input vector and other image preprocessing on the input image, the normalized vector input BP neural network carry on training. When BP network is used in face recognition, each input node of network corresponding to a characteristic of samples, and the output nodes number is equal to the number of categories, an output node corresponds to a class. In the training phase, if the input training samples category punctuation is a, then when the training the desired output is assumed that the $a$-th node is 1 , and the remaining output nodes are 0 . In the recognition phase, when an unknown category sample is applied to the input terminal, inspect each output node corresponding output, and this sample category is determined to have a maximum value of the corresponding to 
the output node of the category. If the maximum value of the distance between the output node and other nodes is small (less than a certain threshold value), determination is refuse to make the judgment. Through competitive selection, to obtain recognition results [9].

\section{Conclusion}

In recent 20 years, researchers in order to improve a variety of insufficient of BP neural network, a large number of studies have been made. Through research can be seen in resolving defects of BP neural network, can use different methods to solve from different aspects. At the same time can see improvements in the excitation function and error function primarily in varying degrees, to improve the convergence speed of the neural network, while the relevant parameters to improve primarily focused on improving network minimization problem [10]. For improved BP neural network, scholars are still making unremitting efforts, hoping to find at all defects can be improved and effective method. Whether attempting to select the appropriate initial weights and thresholds, or change the network structure, from a different perspective, to find a balance point is not a very easy thing to do at all inadequate, people are still doing further research. To perfect for improving BP algorithm, to expand application scope of BP neural network is with great benefits.

\section{References}

[1] L. Huang, "Improvement and Application Research of BP Neural Network Algorithm," Chongqing Normal University, 2008.

[2] Y. K. Li, "Analysis and Improvement Applications of BP Neural Network," Anhui University of Science and Technology, 2012.

[3] Baidu Library, "BP Algorithm," http://wenku.baidu.com/link?url=WfYuJiJ920n7SEGan01p8YRUigmHNchXHa1t7iKdeGLrHv GNShAuVTKps-LEZXiUJBYIsA29qNHQ9s8D5VIvumUWP4CKpUdA39ENSb3rmA, 2014-11-30.

[4] T. S. Liu, "The Research and Application on BP Neural Network Improvement," Northeast Agricultural University, 2011.

[5] H. W. Fan, "Improved BP Algorithm and Its Application in Detection Pavement Crack," Journal of Chang ' an University (Natural Science Edition), vol. 30, no. 1, pp.46-53, 2010.

[6] W. W. Sun, "Study on Improved Algorithm and Application of BP Neural Network," Chongqing University, 2009.

[7] H. Yu, W. Q. Wu, L. Cao, "The Improves on the Standard BP Algorithm and their Use," Computer Knowledge and Technology, vol. 5, no. 19, pp.5256-5258, 2009.

[8] Y. M. Li, "The Study of BP Learning Algorithm of Artificial Neural Network and Its Application in Face Recognition," Shandong University, 2012.

[9] S. Wang, Y. Zhang, R. X. Lv, "BP Neural Network Algorithm Improvement and Application," Computer Knowledge and Technology, vol. 5, no. 4, pp.933-935, 2009.

[10] Q. Y. Lu, X. F. Tong, "Analyses of Improvement Algorithms of BP Neural Network," Computer Engineering and Design, vol. 28, no. 3, pp.648-650, 2007.

[11] M. Xia, "The Research on Improving Generalization of BP Neural Network," Taiyuan University of Science and Technology, 2009.

[12] L. H. Jia, X. R. Zhang, "Analysis and Improvements of BP Algorithm," Computer Technology and Development, vol. 16, no. 10, pp.101-103, 2006. 